\title{
Qualitåtsmanagement der frühen klinischen Behandlung schwerverletzter Patienten
}

ährend in der Industrie Qualitätsmanagement-Systeme allgemein akzeptierte und integrierte Mechanismen zur Verbesserung der Unternehmungsleistung darstellen, finden diese Prinzipien nur zögerlich Eingang in die Medizin. Unter den gesetzlichen Vorgaben des Gesundheitsstrukturgesetzes, aber auch unter wachsenden wirtschaftlichen Zwängen bei gleichzeitig immer größer werdendem Anspruchdenken der Patienten gerät die Medizin unter zunehmenden Druck, qualitätssichernde Maßnahmen nachzuweisen und gleichzeitig darauf aufbauend unter medizinischen und ökonomischen $\mathrm{Ge}$ sichtspunkten eine Optimierung der Versorgungsabläufe zu erzielen. Grundlage eines derartigen Qualitätsmanagements ist die Qualitätssicherung mit den, von A. DONABEDIAN 1988 formulierten Komponenten der Prozess-, Struktur- und Ergebnisqualität. Die Prozessqualität beinhaltet den für den Arzt zunächst im Vordergrund stehenden Untersuchungs- und Behandlungsablauf, wobei dies bisher durch Ausbildung, Erfahrung und Routine letztendlich zu standardisierten Vorgehensweise geführt hatte. Um jedoch regelhafte, nachprüfbare und objektiv weiter vermittelbare Abläufe zu gewährleisten, ist die Erstellung von definierten Protokollen erforderlich, wie diese in den letzten Jahren durch die Formulierung von „Leitlinien"verwirktlicht werden konnten. Derartige Leitlinien, welche als Protokolle, aber auch als Algorithmen formuliert werden, gewährleisten für eine große Zahl aller $\mathrm{Pa}$ tienten einen regelhaften Ablauf, ohne jedoch immer für jede individuelle Situation eine verbindliche Entscheidungsrichtlinie darstellen zu können. Für die Qualitätssicherung bedeuten diese Protokolle darüber hinaus eine ganz wesentliche Grundlage, um mit einer entsprechenden Dokumentation im Sinne eines Ist-Soll-Wert-Vergleiches nach möglichen Abweichungen zu fahnden und ggf. daraus ableitend verbessernde Maßnahmen zu implementieren. Die Strukturqualität beinhaltet die apparativen und personellen Voraussetzungen sowie darauf aufbauend die Organisation der Abläufe. Schließlich gilt es, die Behandlungsleistungen zu objektivieren im Sinne der Ergebnisqualität. Hierbei werden auf der einen Seite zunehmend betriebswirtschaftliche Aspekte angeführt, auf der anderen Seite muß aber weiterhin der medizinische Verlauf mit Beurteilungs-Kriterien wie Letalität, Invalidität, Wiedererlangung der Arbeitsfähigkeit und letztendlich der erreichten Lebensqualität im Vordergrund stehen.

Aufbauend auf den dargestellten qualitätssichernden Komponenten bedeutet das Qualitätsmanagement im zweiten Schritt die Einführung qualitätsverbessernder Maßnahmen. Dazu ist es erforderlich, aufbauend auf den Erkenntnissen und Analysen der Qualitätssicherung durch entsprechende Rückkopplungsschleifen innerhalb der einzelnen Aspekte Verbesserungen zu erzielen. Dabei hat sich in der Industrie bestens bewährt, in diese Prozesse nicht nur die Führungsebene sondern sämtliche Mitarbeiter zu integrieren. Dies geschieht in entsprechenden Qualitätszirkeln, in denen gemeinsam die Dokumentation und Datenanalyse besprochen und nach weiterführenden verbessernden Maßnahmen gesucht werden soll.

Für die Schwerstverletzten-Versorgung wurde zum einen seit den 80 er Jahren sowohl in der deutschsprachigen, als auch in der anglo-amerikanischen Literatur die erforderliche Strukturqualität definiert und darauf aufbauend der Begriff „Traumazentrum" geprägt. Bezüglich der Prozessqualität war seit den 70 er Jahren ein abgestuftes Behandlungsvorgehen propagiert und für Einzelaspekte Algorithmen entwickelt worden. Anfang der go er Jahre wurde von unserer Arbeitsgruppe ein zeitund prioritätenorientierter Algorithmus eingeführt, welcher sämtliche Aspekte der Polytraumabehandlung bei Klinikaufnahme erfaßt. Dieses Konzept wurde inzwischen vielerorts aufgenommen und modifiziert veröffentlicht. Bezüglich der Ergebnisqualität konnte über die Jahre in einer Vielzahl von Publikationen eine kontinuierliche Verbesserung des Behandlungsergebnisses, insbesondere mit einem drastischen Rückgang des posttraumatischen Multiorganversagens und der daraus resultierenden Letalität aufgezeigt werden. Mit der Gründung der „Arbeitsgemeinschaft Polytrauma"der Deutschen Gesellschaft für Unfallchirurgie und des damit etablierten Traumaregisters wurden in Deutschland erstmals die Grundlagen für ein externes Qualitätsmanagement der Schwerstverletztenversorgung geschaffen. Aufbauend auf dieser Form der externen Qualitätskontrolle müssen klinikintern qualitätsverbessernde Maßnahmen implementiert werden, was an zwei eigenen Patientenkollektiven demonstriert werden kann.

Qualitätssicherung, -kontrolle und -management dürfen keinesfalls Politikern, Krankenhausträgern oder Kostenträgern überlassen werden, sondern müssen ausschließlich in ärztlicher Hand bleiben. Vor diesem Hintergrund ist es für die Zukunft für jeden, mit der Behandlung Schwerstverletzter befaßten Kliniker eine unverzichtbare Aufgabe, zum einen mit den Mechanismen der Qualitätssicherung vertraut $z$ u sein und zum anderen aktiv Qualitätsmanagement $z u$ betreiben.
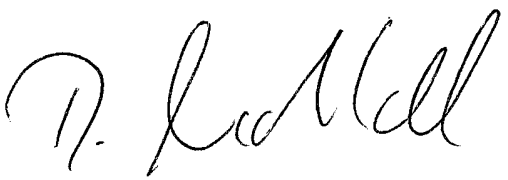

D. Nast-Kolb 